DYSPEPSIA

\title{
Dyspeptic patients with visceral hypersensitivity: sensitisation of pain specific or multimodal pathways?
}

\author{
J Vandenberghe, R Vos, P Persoons, K Demyttenaere, J Janssens, J Tack
}

Gut 2005;54:914-919. doi: 10.1136/gut.2004.052605

See end of article for authors' affiliations

Correspondence to:

Correspondence to:
Dr J Tack, Department of Internal Medicine, Division of Gastroenterology,

University Hospital

Gasthuisberg, Herestraat

49, B-3000 Leuven,

Belgium; Jan.Tack@

med.kuleuven.ac.be

Revised version received 17 February 2005

Accepted for publication

18 February 2005
Background and aims: Patients with functional dyspepsia who have hypersensitivity to gastric distension have more prevalent pain, suggesting the presence of hyperalgesia. It is unclear whether this reflects activation of pain specific afferent pathways or multimodal afferent pathways that also mediate nonpainful sensations. In the former case, hyperalgesia should occur when intensity of non-painful sensations is still low. The aim of the study was to analyse whether the symptom profile during gastric dissentions in functional dyspepsia patients with hyperalgesia reflects sensitisation of pain specific or multimodal pathways.

Methods: Forty eight consecutive dyspeptic patients (35 female) underwent gastric sensitivity testing with a barostat balloon using a double random staircase protocol. At the end of every distending step, patients scored perception of upper abdominal sensations on a graphic 0-6 rating scale and completed visual analogue scales (VAS 0-100 mm) for pain, nausea, satiety, and fullness. The end point was a rating scale of 5 or more.

Results: Hypersensitivity was present in 20 patients (40\%); gastric compliance did not differ between normo- and hypersensitive patients. At maximal distension (score 5 or more), hypersensitive patients had significantly lower distending pressures and intra-balloon volumes, but similar VAS scores for pain, nausea, satiety, and fullness compared with normosensitive patients. In both normosensitive and hypersensitive patients, elevation of pain VAS scores with increasing distending pressures paralleled the elevation in VAS scores for nausea, satiety, and fullness.

Conclusions: Hypersensitive dyspeptic patients reach the same intensity of painful and non-painful sensations as normosensitive patients but at lower distending pressures. Hyperalgesia occurs in hypersensitive dyspeptic patients at distending pressures that also induce intense non-painful sensations. These findings argue against isolated upregulation of pain specific afferents in functional dyspepsia patients with visceral hypersensitivity.
F unctional dyspepsia is a clinical syndrome defined by chronic or recurrent upper abdominal symptoms without an identifiable cause by conventional diagnostic means. ${ }^{1}$ The symptom complex is often related to feeding and includes symptoms of epigastric pain, bloating, early satiety, fullness, epigastric burning, belching, nausea, and vomiting. ${ }^{1}$ Recent studies indicate that functional dyspepsia is a heterogeneous disorder in which different underlying pathophysiological disturbances are associated with specific symptom patterns. ${ }^{2-6}$ During the last decade it has been suggested that visceral hypersensitivity might be major pathophysiological mechanism in functional gastrointestinal disorders. ${ }^{78}$ Gastric barostat studies have confirmed that, as a group, patients with functional dyspepsia have lower thresholds for first perception and for discomfort or pain during balloon distension of the proximal stomach. ${ }^{5-11}$ Hypersensitivity to gastric distension, defined as perception or discomfort thresholds outside the normal range, is found in a subset of patients with functional dyspepsia but not in patients with organic causes of dyspepsia. ${ }^{12}$

Patients with hypersensitivity to gastric distension have more prevalent symptoms of epigastric pain. ${ }^{513}$ During gastric balloon distension, patients with visceral hypersensitivity experience pain at levels of distension that are not painful under normal circumstances, ${ }^{9-11}$ suggesting the presence of visceral hyperalgesia. ${ }^{8}$ These observations indicate sensitisation at one level or another of afferent pathways that convey information from the stomach to the central nervous system. According to the neurophysiological theory of pain, pain can be encoded by activation of high threshold nociceptive pathways or by intense stimulation of low threshold multimodal pathways (fig 1A). ${ }^{14}$ In the gastrointestinal tract, animal studies have demonstrated spinal afferents that respond to both noxious and non-noxious events with different intensity of discharge. ${ }^{14-18}$ However, high threshold mechanoreceptors, thought to act as mechanonociceptors, were also reported. ${ }^{14-18}$ In theory, hyperalgesia could be related to sensitisation of nociceptive pathways, in which case the intensity of non-painful sensations would remain unaltered (fig 1B). Alternatively, hyperalgesia could also occur because of sensitisation of multimodal pathways, in which case the intensity of non-painful sensations should also be increased (fig lC). Finally, hyperalgesia could be due to a combined sensitisation of high threshold nociceptive pathways and low threshold multimodal pathways (fig 1B, C), which would also result in an increased intensity of nonpainful sensations.

The aim of the present study was to investigate whether gastric hyperalgesia is related to sensitisation of pain specific or multimodal afferent pathways. To differentiate between both, we analysed the intensity profile of painful and non-painful sensations during gastric distension in dyspeptic patients with hypersensitivity to gastric distension and in dyspeptic patients with normal sensitivity to distension. In the case of isolated sensitisation of nociceptive pathways, only the intensity of painful sensations should

Abbreviations: VAS, visual analogue scale; MDP, minimal distending pressure 

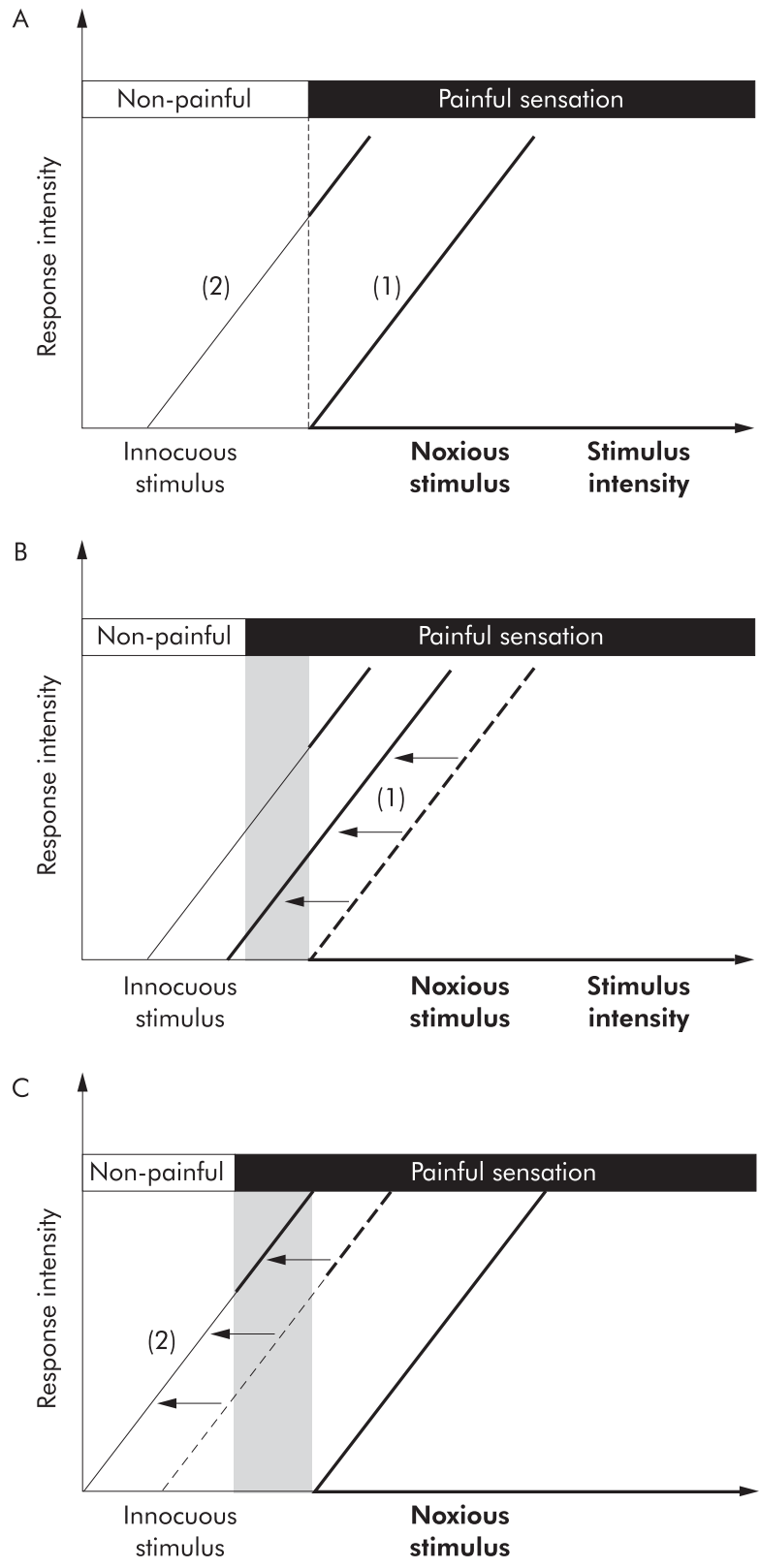

Figure 1 Putative pathways involved in perception of painful and nonpainful gastric stimuli. (A) Model of the normal physiology of afferent pathways. (B, C) Models of the pathophysiology of hyperalgesia, a pathological condition characterised by innocuous stimuli causing painful sensations (reflected by the shaded area). (A). Pain can be encoded by activation of high threshold nociceptive pathways (1) and/or by intense stimulation of low threshold multimodal pathways (2). Both pathways show a higher response ( $Y$ axis) with increasing stimulus intensity. Only noxious stimuli result in painful sensations. (B) Hyperalgesia can be related to sensitisation of nociceptive pathways (1), in which case the intensity of non-painful sensations would remain unaltered (isolated hyperalgesia). (C) Alternatively, hyperalgesia could also occur because of sensitisation of multimodal pathways (2), in which case the intensity of non-painful sensations should also be increased (hyperalgesia combined with general hypersensitivity).

be significantly higher in patients with hypersensitivity at a given stimulus intensity. In the case of sensitisation of multimodal pathways or of both pathways, both painful and non-painful sensations should be significantly higher in patients with hypersensitivity at a given stimulus intensity.

\section{MATERIALS AND METHODS}

\section{Study subjects}

Consecutive patients with functional dyspepsia were recruited to the study. Patients presented to the motility outpatient clinic because of meal related epigastric symptoms, and all underwent careful history taking and clinical examination, upper gastrointestinal endoscopy, routine biochemistry, and upper abdominal ultrasound. Inclusion criteria were the presence of dyspeptic symptoms for at least 12 weeks in the last 12 months, in the absence of organic, systemic, or metabolic disease. Dyspeptic symptoms had to be present for at least three days per week, with two or more symptoms scored as relevant or severe on the symptom questionnaire (see below). Exclusion criteria were the presence of oesophagitis, gastric atrophy, or erosive gastroduodenal lesions on endoscopy, heartburn as a predominant symptom, a history of peptic ulcer, major abdominal surgery, underlying psychiatric illness, and the use of non-steroidal anti-inflammatory drugs, steroids, or drugs affecting gastric acid secretion. During upper gastrointestinal endoscopy, biopsies were taken from the antrum and corpus to stain with cresyl violet for the presence of Helicobacter pylori. A psychiatrist ruled out anorexia nervosa in patients with weight loss in excess of $5 \%$ of initial body weight. All patients were also screened for major depression or anxiety states, and those with major psychiatric morbidity were excluded. All drugs potentially affecting gastrointestinal motility or sensitivity were discontinued at least one week prior to the barostat study.

Informed consent was obtained from each participant. The protocol was previously approved by the ethics committee of the university hospital.

\section{Symptom questionnaire}

Each patient completed a dyspepsia questionnaire, as reported previously. ${ }^{4-6}$ The patient was asked to grade the intensity $(0-3 ; \quad 0=$ absent, $1=$ mild, 2 = relevant, and $3=$ severe, interfering with daily activities) of eight different symptoms (epigastric pain, bloating, postprandial fullness, early satiety, nausea, vomiting, belching, and epigastric burning) over the last three months. Also, the amount of weight lost since the onset of symptoms was noted.

\section{Gastric barostat studies}

Following an overnight fast of at least 12 hours, a double lumen polyvinyl tube (Salem sump tube $14 \mathrm{Ch}$; Sherwood Medical, Petit Rechain, Belgium) with an adherent plastic bag ( $1200 \mathrm{ml}$ capacity; $17 \mathrm{~cm}$ maximal diameter) finely folded, was introduced through the mouth and secured to the subject's chin with adhesive tape. The position of the bag in the gastric fundus was checked fluoroscopically. The polyvinyl tube was then connected to a programmable barostat device (Synectics Visceral Stimulator, Stockholm, Sweden). To unfold the bag it was inflated with a fixed volume of $300 \mathrm{ml}$ of air for two minutes with the study subject in a recumbent position, and again deflated completely. Subjects were then positioned in a comfortable sitting position with the knees bent $\left(80^{\circ}\right)$ and the trunk upright in a specifically designed bed.

After a 30 minute adaptation period, minimal distending pressure (MDP) was first determined by increasing intraballoon pressure by $1 \mathrm{~mm} \mathrm{Hg}$ every three minutes until a volume of $30 \mathrm{ml}$ or more was reached. ${ }^{45}{ }^{19}$ This pressure level equilibrates with intra-abdominal pressure. Subsequently, isobaric distensions were performed using a double random staircase protocol with stepwise increments of $2 \mathrm{~mm} \mathrm{Hg}$ starting from MDP, each lasting for two minutes, while the corresponding intra-balloon volume was recorded. We 
previously established that sensitivity thresholds in patients with functional dyspepsia are reproducible. ${ }^{19}$

During the last 30 seconds of every distending step, subjects were instructed to score their perception of upper abdominal sensations using a graphic rating scale that combined verbal descriptors on a scale graded $0-6 .{ }^{45}{ }^{19}$ The end point of each sequence of distensions was established at an intra-balloon volume of $1000 \mathrm{ml}$ or when subjects reported discomfort (score 5) or pain (score 6). In addition, also during the last 30 seconds of each pressure step, subjects rated the sensations of epigastric pain, fullness, nausea, and satiety on a visual analogue scale (VAS). The VAS consisted of a $100 \mathrm{~mm}$ long line with $0 \mathrm{~mm}$ indicating "no sensation" and $100 \mathrm{~mm}$ indicating "the strongest sensation ever felt".

A 30 minutes adaptation period with the bag completely deflated was then allowed, following which the pressure level was set at $\mathrm{MDP}+2 \mathrm{mmHg}$ for 90 minutes for measurement of gastric tone and phasic contractile activity. After 30 minutes a standardised liquid meal was given $(200 \mathrm{ml}, 300 \mathrm{kcal} ; 13 \%$ proteins, $48 \%$ carbohydrates, 39\% lipids; Nutridrink, Nutricia, Bornem, Belgium) and measurements continued for another 60 minutes.

\section{Gastric emptying studies}

Gastric emptying for solids was measured in patients using the previously validated ${ }^{14} \mathrm{C}$ octanoic breath test. ${ }^{20}$ Briefly, all studies were carried out in the morning after an overnight fast. The test meal consisted of $60 \mathrm{~g}$ of white bread, 1 egg, the yolk of which was doped with $74 \mathrm{kBq}$ of ${ }^{14} \mathrm{C}$ octanoic acid sodium salt, and $300 \mathrm{ml}$ of water. Breath samples were taken before the meal and at 15 minutes intervals for a period of 240 minutes postprandially. Gastric half emptying time $(\mathrm{tl} / 2)$ was calculated as previously described..$^{20}$

\section{Data analysis}

For each two minute isobaric distending period, intrabag volume was calculated by averaging the recording. Perception threshold was defined as the first level of pressure relative to MDP and the corresponding volume that evoked a perception score of 1 or more. Discomfort threshold was defined as the first level of pressure relative to MDP and the corresponding volume that provoked a score of 5 or more.

Pressure-volume and pressure-perception curves were obtained from the stepwise distensions. As reported previously, a linear regression model provided the best fit. ${ }^{21}$ Gastric compliance was calculated as the slope and the intercept of the pressure-volume curve obtained during the first three steps of isobaric distensions.

Gastric tone before and after administration of the meal was measured by calculation of mean balloon volumes for consecutive five minute intervals. Meal induced gastric relaxation was quantified as the difference between the average volumes during 30 minutes before 60 minutes after the meal.
Table 2 Demographic features in 48 dyspeptic patients with or without hypersensitivity to gastric distension

\begin{tabular}{lll}
\hline & $\begin{array}{l}\text { Normal } \\
\text { sensitivity }\end{array}$ & Hypersensitivity \\
\hline Age (y) & $44(2)$ & $31(3)^{*}$ \\
Female sex (\%) & $20(71 \%)$ & $15(75 \%)$ \\
Body mass index $\left(\mathrm{kg} / \mathrm{m}^{2}\right)$ & $20.5(0.6)$ & $21.7(0.7)$ \\
MDP (mm Hg) & $7.3(0.5)$ & $5.6(0.4)^{*}$ \\
Hp positive (\%) & $2(7)$ & $1(5)$ \\
Impaired accommodation (\%) & $8(29)$ & $4(20)$ \\
Delayed gastric emptying (\%) & $7(25)$ & $4(19)$ \\
\hline MDP, minimal distending pressure; Hp, Helicobacter pylori. \\
*p<0.05 compared with patients with normal sensitivity to gastric \\
distension.
\end{tabular}

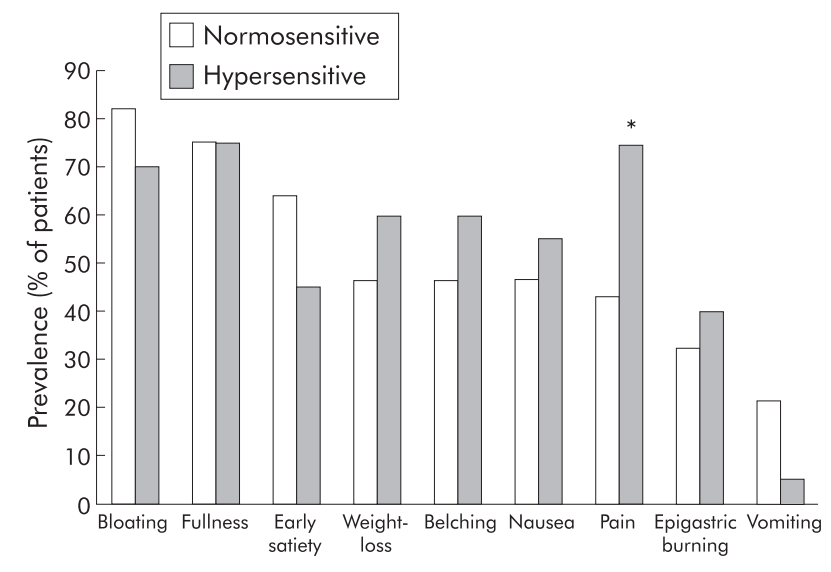

Figure 2 Dyspepsia symptoms in 48 functional dyspepsia patients. Number of patients grading individual symptoms as moderate or severe (score $>1$ ) in the subgroups with normal sensitivity or hypersensitivity to gastric distension. Postprandial pain was significantly more prevalent in patients with hypersensitivity to gastric distension ( ${ }^{*} \mathrm{p}<0.05$ ).

\section{Statistical analysis}

By using previously found normal ranges for healthy volunteers in our laboratory, we defined patients with impaired accommodation (meal induced gastric relaxation $<64 \mathrm{ml}$ ), hypersensitivity to gastric distension (discomfort threshold $<6.6 \mathrm{~mm} \mathrm{Hg}$ above MDP), and delayed gastric emptying for solids $(\mathrm{tl} / 2>109 \mathrm{~min}){ }^{4-6}$ Patients were subdivided into those with normal sensitivity and hypersensitivity to gastric distension. Demographic characteristics, MDP, and gastric compliance were compared between both groups using the Student's $t$ test and $\chi^{2}$ testing. VAS scores for individual symptoms were compared within and between patients groups using two way ANOVA. Pearson's linear correlation analysis was used to study correlations between

\begin{tabular}{|c|c|c|c|c|}
\hline & 0 (Absent) & 1 (Mild) & 2 (Moderate) & 3 (Severe) \\
\hline Postprandial fullness & $3(6)$ & $9(19)$ & $25(52)$ & $11(23)$ \\
\hline Bloating & $4(8)$ & $7(15)$ & $28(58)$ & $9(19)$ \\
\hline Nausea & $11(23)$ & $13(27)$ & $18(38)$ & $6(12)$ \\
\hline Epigastric pain & $9(19)$ & $12(25)$ & $21(44)$ & $6(12)$ \\
\hline Early satiety & $15(31)$ & $6(13)$ & $21(44)$ & $6(13)$ \\
\hline Belching & $10(21)$ & $13(27)$ & $22(46)$ & $3(6)$ \\
\hline Epigastric burning & $20(42)$ & $11(23)$ & $12(25)$ & $5(10)$ \\
\hline Vomiting & $33(69)$ & $8(17)$ & $5(10)$ & $2(4)$ \\
\hline
\end{tabular}



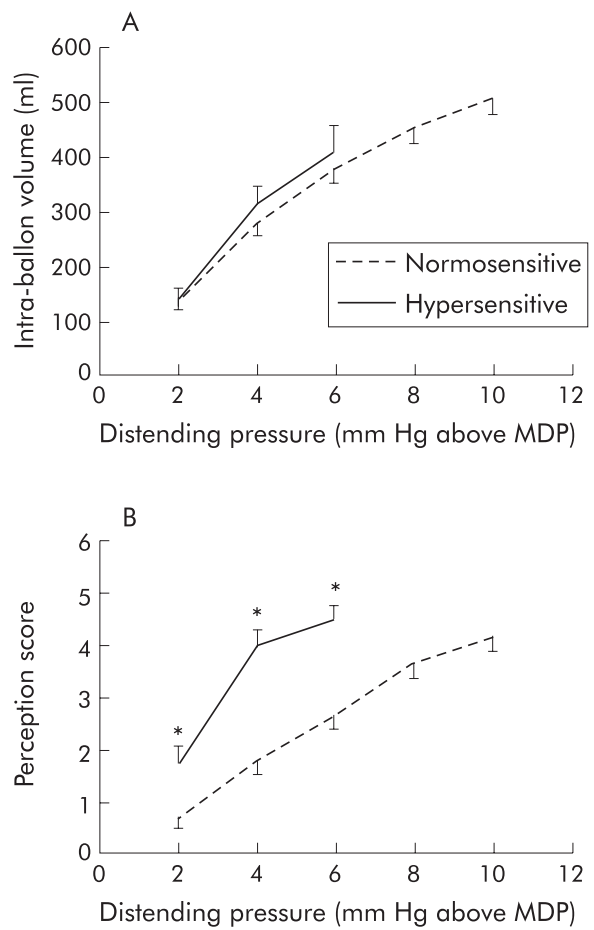

Figure 3 Responses during isobaric gastric distensions in 48 patients with functional dyspepsia. (A) Pressure-volume relationship in patients with hypersensitivity and normal sensitivity to gastric distension. (B) Pressure-perception score relationship in patients with hypersensitivity and normal sensitivity to gastric distension. ${ }^{*} \mathrm{p}<0.05$ compared with patients with normal sensitivity to gastric distension. Note that three of the hypersensitive patients reached a score of 5 or 6 at a distending pressure of $2 \mathrm{~mm} \mathrm{Hg}$ above the minimal distending pressure (MDP), seven at $4 \mathrm{~mm} \mathrm{Hg}$, and 10 at $6 \mathrm{~mm} \mathrm{Hg}$. Similarly, for normosensitive patients, data are only shown up to the distending pressure where a value was available for more than $50 \%$ of patients.

VAS scores for different individual symptoms in each patient group.

Differences were considered significant at the $5 \%$ level. Data are presented as mean (SEM). Bonferroni's correction for multiple comparisons was applied. The study was calculated to have an $85 \%$ power to detect a $30 \%$ difference in symptom intensity between pain and non-painful sensations.

\section{RESULTS}

\section{Patient descriptives}

Forty eight consecutive functional dyspepsia patients ( 13 men and 35 women; mean age 38 (2) years) participated in the study. Table 1 summarises the grading of dyspeptic symptoms in the patient group. Postprandial fullness and bloating were the most prevalent symptoms, present in $94 \%$ and $92 \%$ of patients, respectively. Epigastric pain $(81 \%)$, belching $(79 \%)$, nausea $(77 \%)$, and early satiety $(69 \%)$ were also frequently reported. Vomiting and epigastric burning sensation were present in $31 \%$ and $58 \%$ of patients, respectively. Weight loss in excess of 5\% was present in 25 patients $(52 \%)$. H pylori was demonstrated on gastric biopsies in three patients $(6 \%)$. Delayed gastric emptying was present in 11 patients $(22 \%)$. Impaired accommodation to a meal was present in 12 patients $(25 \%)$.

\section{Characteristics of patients with or without hypersensitivity to gastric distension}

Hypersensitivity to gastric distension was present in 20 patients (42\%); gastric sensitivity was normal in the other 28 patients. As previously reported, ${ }^{5}$ patients with hypersensitivity to gastric distension were significantly younger and had a lower MDP compared with patients with normal sensitivity. The sex distribution and prevalence of Helicobacter infection did not differ between the groups (table 2). The prevalence of relevant or severe pain was significantly higher in hypersensitive patients $(15 / 20 \vee 12 / 28 ; p<0.05)$; the prevalence of other symptoms did not differ between the groups of patients (fig 2).

\section{Gastric compliance and distension end points in patients with or without hypersensitivity to gastric distension}

In both patients groups, gastric distension with progressively higher set pressures produced progressively larger intraballoon volumes. Gastric compliance did not differ between the groups but corresponding symptom scores for the same distending pressure were significantly higher in hypersensitive patients (fig 3). The maximum distending pressure and corresponding intra-balloon volume were significantly lower in hypersensitive patients compared with normosensitive patients (table 3 ). At the maximum distending pressure, the intensity of painful and non-painful symptoms did not differ significantly between the groups (table 3 ).

\section{Painful and non-painful sensations during gastric distension in patients with or without hypersensitivity to gastric distension}

In patients with normal sensitivity to gastric distension, progressively higher set pressures produced progressively higher intensity scores of all symptoms assessed. Intensity scores did not differ between painful and any of the nonpainful symptoms (fig 4). VAS symptom intensity scores between painful and non-painful symptoms were only weakly correlated (all $r<0.34,0.003<\mathrm{p}<0.05$ ).

In patients with hypersensitivity to gastric distension, progressively higher set pressures produced progressively

Table 3 Gastric compliance and distension end points in 48 dyspeptic patients with or without hypersensitivity to gastric distension

\begin{tabular}{|c|c|c|}
\hline & Normal sensitivity & Hypersensitivity \\
\hline Slope of gastric compliance curve $(\mathrm{ml} / \mathrm{mm} \mathrm{Hg})$ & $58(5)$ & $72(8)$ \\
\hline Intercept of gastric compliance curve (ml) & $33(15)$ & $3(27)$ \\
\hline Maximal distending pressure ( $\mathrm{mm} \mathrm{Hg}$ above MDP) & $10.2(0.6)$ & $4.7(0.3)^{\star *}$ \\
\hline Corresponding intra-balloon volume (ml) & $530(35)$ & $342(41)^{* *}$ \\
\hline Corresponding perception score & $5.2(0.1)$ & $5.2(0.1)$ \\
\hline Corresponding pain intensity $(\mathrm{mm})$ & $45(7)$ & $57(8)$ \\
\hline Corresponding nausea intensity $(\mathrm{mm})$ & $47(7)$ & $52(8)$ \\
\hline Corresponding satiety intensity $(\mathrm{mm})$ & $46(7)$ & $60(8)$ \\
\hline Corresponding fullness intensity $(\mathrm{mm})$ & $56(7)$ & $62(8)$ \\
\hline
\end{tabular}



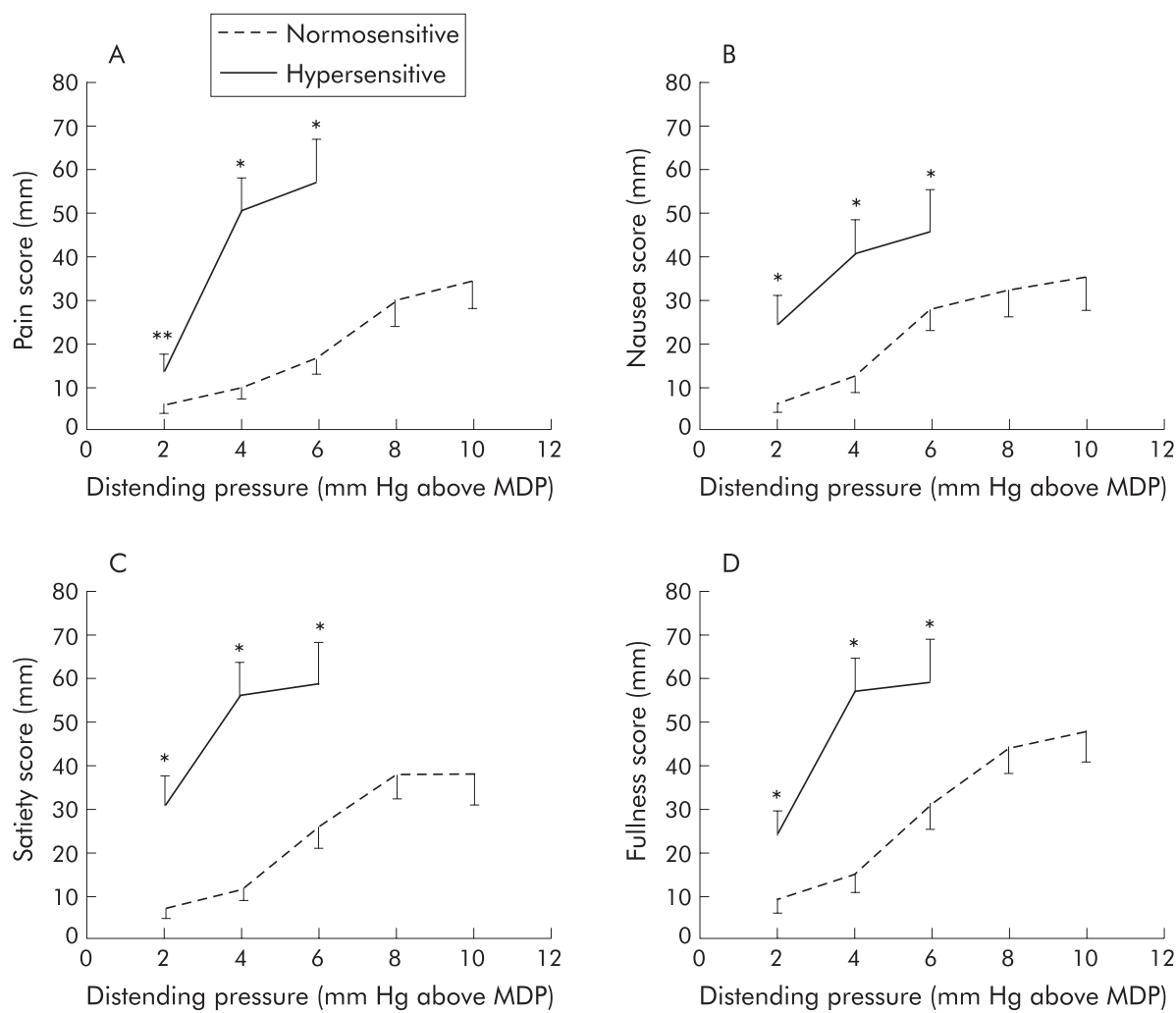

Figure 4 Symptom severities on visual analogue scales during isobaric gastric distensions in 48 patients with functional dyspepsia. (A) Intensities for pain in patients with hypersensitivity and normal sensitivity to gastric distension. (B) Intensities for nausea in patients with hypersensitivity and normal sensitivity to gastric distension. (C) Intensities for satiety in patients with hypersensitivity and normal sensitivity to gastric distension. (D) Intensities for fullness in patients with hypersensitivity and normal sensitivity to gastric distension. ${ }^{*} p<0.05,{ }^{* *} p=0.07$ compared with patients with normal sensitivity to gastric distension. Note that three of the hypersensitive patients reached a score of 5 or 6 at a distending pressure of $2 \mathrm{~mm} \mathrm{Hg}$ above minimal distending pressure (MDP), seven at $4 \mathrm{~mm} \mathrm{Hg}$, and 10 at $6 \mathrm{~mm} \mathrm{Hg}$. Similarly, for normosensitive patients, data are only shown up to the distending pressure where a value was available for more than $50 \%$ of patients.

higher intensity scores of all symptoms assessed. The intensity scores did not differ between painful and any of the non-painful symptoms. In hypersensitive patients, excellent correlations were found between VAS symptom intensity scores for pain and fullness $(r=0.79, \mathrm{p}<0.0001)$ and satiety $(r=0.73, \mathrm{p}<0.0001)$. The correlation between nausea and pain VAS scores was weaker $(r=0.41, \mathrm{p}=0.02)$. At any given distending pressure, scores for all symptoms (both painful and non-painful) were significantly higher in hypersensitive patients compared with normosensitive patients (fig 4).

\section{DISCUSSION}

For more than a decade, visceral hypersensitivity has been considered a major pathophysiological factor in functional gastrointestinal disorders. ${ }^{78}$ In functional dyspepsia, using a gastric barostat, several investigators demonstrated lower sensory thresholds during balloon distension of the proximal stomach compared with healthy volunteers. ${ }^{\text {9-12 }}$ Hypersensitivity to gastric distension seems to be a feature of functional but not organic dyspepsia ${ }^{12}$ and is also present in dyspeptic subjects who are not health care seekers, implying that visceral hypersensitivity is not solely an expression of referral bias or personality factors. ${ }^{22}$ The mechanism behind hypersensitivity to gastric balloon distension in functional dyspepsia is not altogether clear but an abnormal afferent sensory pathway has been proposed. ${ }^{9}$ The sensory pathways involved in mediating gastric perception in health and disease have not been fully characterised. A better understanding of the characteristics of these pathways is likely to enhance pathophysiological knowledge and may lead to more optimal therapeutic approaches.

Patients with functional dyspepsia may report pain as well as a variety of non-painful symptoms, often referred to as discomfort. ${ }^{123}{ }^{24}$ Gastric balloon distension can elicit both painful and non-painful sensations, in health as well as in functional dyspepsia. ${ }^{9-12}$ Patients with hypersensitivity to gastric distension have more prevalent symptoms of epigastric pain, ${ }^{513}$ suggesting the presence of visceral hyperalgesia. $^{78}$ It is unclear whether this reflects selective sensitisation for painful sensations or whether the sensitivity for nonpainful stimuli is also enhanced in patients with visceral hypersensitivity.

In the present study, we confirmed the association of hypersensitivity to gastric distension with more prevalent symptoms of pain. We observed that, during gastric balloon distension, patients with hypersensitivity to gastric distension had higher pain scores at a given stimulus intensity than patients with normal sensitivity. In addition, we observed that scores for non-painful sensations of fullness, nausea, and satiety at a given stimulus intensity were also significantly higher in patients with hypersensitivity compared with patients with normal sensitivity. In both normosensitive and hypersensitive dyspeptic patients, the increase in intensity scores for pain paralleled the increase in intensity scores for the non-painful sensations of nausea, satiety, and fullness. As gastric compliance did not differ between the groups of patients, differences in sensory ratings do reflect alterations in perception pathways, and not gastric wall properties.

In general, patients seemed able to distinguish between the four different symptoms that were assessed during gastric 
balloon distensions. This is supported by the absence of a significant correlation between intensities of the different symptoms in normosensitive patients. In hypersensitive patients, pain scores were closely correlated with fullness and satiety scores, but nausea scores showed a poor correlation with pain scores.

In hypersensitive patients, hyperalgesia occurred at distending pressures that also induced intense non-painful sensations. Furthermore, hypersensitive patients reported the same type of symptoms and reached the same intensity of non-painful sensations as normosensitive patients, but at lower distending pressures. These findings argue against isolated upregulation of pain specific afferent pathways in functional dyspepsia with visceral hyperalgesia. They are compatible with upregulation of multimodal afferent pathways, and this is further supported by the significant correlations between pain and fullness and satiety scores in hypersensitive patients only. Sensitisation of both types of afferent pathways seems less likely as it assumes that two different sensory systems underwent comparable upregulation, but cannot be ruled out entirely. We also cannot exclude the existence of even higher threshold pain specific pathways that were not activated by the current balloon distension paradigm.

Sensitisation to gastric distension may occur at the level of peripheral afferents but also at the level of the central nervous system. ${ }^{8}{ }^{14}$ Furthermore, although the double random staircase protocol aimed at minimising expectation based response bias, a number of other factors such as hypervigilance and anxiety may have contributed to the intensity ratings during gastric distension..$^{1325}$ The mechanisms and anatomical levels involved in this upregulation of multimodal, with or without involvement of pain specific afferent, pathways could not be addressed by the present study and remains to be elucidated. However, the close relationship between painful and non-painful symptoms in hypersensitive patients suggests that therapeutic interventions aimed at decreasing hyperalgesia and pain would potentially also decrease non-painful dyspeptic symptoms in these patients. Furthermore, as pain and non-painful sensations showed parallel increments in both normosensitive and hypersensitive patients, this observation questions the subdivision in pain predominant and discomfort predominant dyspeptic patients, as proposed in the Rome II classification. ${ }^{1}$

In summary, we did not find evidence in favour of isolated upregulation of pain specific afferents in functional dyspepsia patients with visceral hyperalgesia. Hyperalgesia occurs in hypersensitive dyspeptic patients at distending pressures that also induce intense non-painful sensations. Hypersensitive dyspeptic patients reached the same intensity of painful and non-painful sensations as normosensitive patients but at lower distending pressures. The mechanisms and anatomical levels involved in the upregulation of presumably multimodal afferent pathways remain to be elucidated.

\section{Authors' affiliations}

J Vandenberghe, P Persoons, K Demyttenaere, Department of

Psychiatry, Division of Liaison Psychiatry, Division of Gastroenterology, University Hospital Gasthuisberg, University of Leuven, Leuven, Belgium

R Vos, J Janssens, J Tack, Department of Internal Medicine, Division of Gastroenterology, University Hospital Gasthuisberg, University of Leuven, Leuven, Belgium

Conflict of interest: None declared.

\section{REFERENCES}

1 Talley NJ, Stanghellini V, Heading RC, et al. Functional gastroduodenal disorders. Gut 1999;45(supple II):37-42.

2 Talley NJ. Functional dysypepsia: Should treatment be targeted on disturbed physiology? Aliment Pharmacol Ther 1995;9:107-15.

3 Stanghellini V, Tosetti C, Paternico A, et al. Risk indicators of delayed gastric emptying of solids in patients with functional dyspepsia. Gastroenterology 1996; 110:1036-42.

4 Tack J, Piessevaux H, Coulie B, et al. Role of impaired gastric accommodation to a meal in functional dyspepsia. Gastroenterology 1998; 1 15:1346-52.

5 Tack J, Caenepeel P, Fischler B, et al. Symptoms associated with hypersensitivity to gastric distension in functional dyspepsia. Gastroenterology 2001;121:526-35.

6 Sarnelli G, Caenepeel P, Geypens B, et al. Symptoms associated with impaired gastric emptying of solids and liquids in functional dyspepsia. Am J Gastroenterol 2003;98:783-8.

7 Camilleri M, Coulie B, Tack J. Visceral hypersensitivity: facts, speculations and challenges. Gut 2001;48:125-31.

8 Mayer EA, Gebhart FG. Basic and clinical aspects of visceral hyperalgesia. Gastroenterology 1994; 107:271-93

9 Mearin F, Cucala M, Azpiroz F, et al. The origin of symptoms on the brain-gut axis in functional dyspepsia. Gastroenterology 1991;101:999-1006.

10 Lemann M, Dederding JP, Flourie B, et al. Abnormal perception of visceral pain in response to gastric distension in chronic idiopathic dyspepsia. The irritable stomach syndrome. Dig Dis Sci 1991;36:1249-54.

11 Bradette M, Pare P, Douville P, et al. Visceral perception in health and functional dyspepsia. Crossover study of gastric distensions with placebo and domperidone. Dig Dis Sci 1991;36:52-8.

12 Mertz H, Fullerton S, Naliboff B, et al. Symptoms and visceral perception in severe functional and organic dyspepsia. Gut 1998;42:814-22.

13 Fischler B, Tack J, De Gucht V, et al. Heterogeneity of symptom pattern, psychosocial factors, and pathophysiological mechanisms in severe functional dyspepsia. Gastroenterology 2003;124:903-10.

14 Mayer EA, Raybould HE. Role of visceral afferent mechanisms in functional bowel disorders. Gastroenterology 1990;99:1688-704.

15 Blumberg $H$, Haupt $P$, Jaenig $Z$, et al. Encoding of visceral noxious stimuli in the discharge patterns of visceral afferent fibers from the colon. Pfluegers Arch 1983;398:33-40

16 Cervero F. Sensory innvervation of the viscera: peripheral basis of visceral pain. Physiol Rev 1994;74:95-138.

17 Janig W, Koltzenburg M. On the function of spinal primary afferent fibres supplying colon and urinary bladder. J Auton Nerv Syst 1990;30:S89-96.

18 Sengupta JN, Saha JK, Goyal RK. Stimulus-response function studies of esophageal mechanosensitive nociceptors in sympathetic afferents of opossum. J Neurophysiol 1990;64:796-812.

19 Sarnelli G, Vos R, Cuomo R, et al. Reproducibility of gastric barostat studies in healthy controls and in dyspepetic patients. Am J Gastroenterol $2001 ; 96: 1047-53$

20 Ghoos YF, Maes BD, Geypens BJ, et al. Measurement of gastric emptying rate of solids by means of a carbon labeled octanoic acid breath test. Gastroenterology 1993;104:1640-7.

21 Tack J, Coulie B, Wilmer A, et al. Effect of sumatriptan on gastric fundus tone and on the perception of gastric distension in man. Gut 2000;46:468-73.

22 Holtmann G, Gschossmann J, Neufang-Huber J, et al. Differences in gastric mechanosensory function after repeated ramp distensions in non-consulters with dyspepsia and healthy controls. Gut 2000;47:332-6.

23 Stanghellini V, Tosetti C, Paternico A, et al. Predominant symptoms identify different subgroups in functional dyspepsia. Am J Gastroentero/ 1999;94:2080-5.

24 Stanghellini V. Pain versus discomfort-is differentiation clinically useful? Aliment Pharmacol Ther 2001;15:145-9.

25 Naliboff BD, Munakata J, Fullerton S, et al. Evidence for two distinct perceptual alterations in irritable bowel syndrome. Gut 1997;41:505-12. 